\title{
The Design and Manufacture of Custom Ocular Prostheses (COPs) Based on Computer-Aided Semi- automation Workflow
}

Xin Ye

Affiliated Eye Hospital of Wenzhou Medical College

Jun Wang

Affiliated Eye Hospital of Wenzhou Medical College

Wei Fang

Affiliated Eye Hospital of Wenzhou Medical College

Zhenbin Qian

Affiliated Eye Hospital of Wenzhou Medical College

Lijun Shen ( $\nabla$ slj@mail.eye.ac.cn )

Affiliated Eye Hospital of Wenzhou Medical College

\section{Research Article}

Keywords: Custom ocular prosthesis, Computer-aided design, Reverse engineering, 3D printing

Posted Date: April 19th, 2021

DOI: https://doi.org/10.21203/rs.3.rs-388211/v1

License: (9) This work is licensed under a Creative Commons Attribution 4.0 International License.

Read Full License 


\section{Abstract \\ Background}

It is important to correct the ocular disfigurement by wearing prostheses as soon as possible after healing from the eye enucleation surgery. This study aimed to develop a new computer-aided approach to fabricate custom ocular prostheses (COPs) with higher accuracy and efficiency and to fit different anophthalmia sockets.

\section{Results}

Five patients underwent enucleation surgery from November 2018 to October 2020 were enrolled in the study. The semi-automatic design and fabrication workflow of COPs was based on the conjunctival sac impression, combined with Computer-aided design/Computer-aided manufacture (CAD/CAM) workflow, which involves computer image analysis, reverse engineering (RE), and 3-dimensional (3D) printing technologies. The height of palpebral fissure in the artificial eye group was $0.8000 \pm 0.09529, N=5$. The height of palpebral fissure in the healthy eye group was $0.7660 \pm 0.1147, \mathrm{~N}=5$, and there was no statistical difference between the two groups $(p>0.05)$. The artificial eye in the conjunctival sac had a good effect on the reconstruction of orbital fullness. Both of the vertical activity and horizontal activity of COPs is smaller than that of healthy eyes $(p<0.05)$.

\section{Conclusions}

COPs made by RE and 3D printing technologies can effectively make the COPs suitable for patients' conjunctival sac. This workflow improved the accuracy of the COPs, and also reduced patients' waiting time, which is worthy to be popularized and applied.

\section{Introduction}

It is important to correct the ocular disfigurement by wearing prostheses as soon as possible after healing from the eye enucleation surgery. The need for an artificial eye can satisfied by stock prostheses that come in different sizes, shapes, and colors[1]. Traditional methods to make custom ocular prostheses (COPs) take a long time to complete due to series of complex steps, which strongly depend on the experience of the oculist[2]. However, there still exists unsatisfactory match between COPs and the patients' conjunctival sac in clinical practice, which may cause pain, secretion, and impaired appearance[3]. Thus, it is meaningful to develop a new approach to fabricate COPs with higher accuracy and efficiency and to fit different anophthalmia sockets.

Traditional basic steps of making COPs are as follows[4-6]: (1) Making a paraffin model of the conjunctival sac when it softened after heating; (2) Making a gypsum molding according to the paraffin 
model; (3) Making the opaque resin model: mix the resin with oxygen albendazole powder and resin liquid in proportion, pour into the gypsum model to make the opaque resin model; (4) Coloring: using oily pigment, brush, acrylic binder, and SAP for manual coloring according to that of healthy eye; (5) Cover the colored opaque resin surface with translucent SAP; (6) Try it on and follow-up for 3-6 months, and modify it several times according to the shape of the conjunctival sac until the eye socket is well formed. In this process, the modeling of conjunctival sac is an important stage, but the effect of conjunctival sac modeling is often affected by the personal skill of doctors and the degree of patient cooperation[7]. In addition, the process from the gel membrane to the wax mold which is suitable for the conjunctival sac space of the patient is complicated, time-consuming, and subject to much interference from the subjective factors of the doctor.

With the development RE technologies, a corresponding digital model was constructed from an existing physical object by 3D reconstruction. Based on advanced mathematical model and surface construction theory, reverse engineering (RE) softwares can quickly sort surface point cloud data and automatically generate grids, which can build arbitrary complex accurate surface models and create high-quality 3D models[8]. With its advantages in surface fitting, RE software has widely used in medical research. Therefore, applying the RE technologies to COPs production can improve the visualization and precision of the design process.

$3 \mathrm{D}$ printing technology is very suitable for fabricating an object from a model accurately $[9,10]$. Fused deposition (FDM) technology mainly makes use of the heat melting and adhesion of thermoplastic materials, which are stacked layer by layer under the control of computer, and finally the processed entity is obtained. Under the control of computer, FDM heating head heating the material (ABS, nylon, wax, and other materials) to flow state, and the nozzle parts move along the cross-section contour and filling path at the same time. The material solidified rapidly and adhered layer by layer, thus, bottom-up form a 3D object[11].

This study aimed to develop a computer-aided semi-automatic workflow for designing and manufacturing COPs, which can optimize the shape of the COP and making it more comfortable for wearing. Computer-aided design/Computer- aided manufacture (CAD/CAM) involve computer image analysis, reverse engineering (RE), three-dimensional (3D) printing and other technologies, which may provide new ideas for designing and fabricating COPs. The anatomy of eyes is complex and meticulous, and the designing of 3D model is a difficulty. Moreover, the selection of printing technology needs to be combined with the comprehensive selection of 3D printing materials.

\section{li. Materials And Methods}

\section{A. DATA COLLECTION SPECIFICATIONS}

Five patients underwent enucleation surgery from November 2018 to October 2020. The patients had implants in orbit. Subject inclusion criteria were no conjunctival sac or accompanied by mild 
inflammation, no conjunctival sac granuloma, no eyelid deformity, upper eyelid droop, lower eyelid relaxation, orbital contracture, rabbit eye deformity and other complications.

\section{B. MATERIALS}

Elastomer impression material (Vinylpolysiloxane Impression Material Light Body, the United States I - SiL company). Binocular Blue ray 3D scanner (Shenzhen Nanke 3D Technology Co., LTD). Main performance indicators: Single amplitude measurement range $(\mathrm{mm})$ : $100 \times 75$; Single amplitude measurement accuracy: $0.01 \sim 0.015$; Sampling point distance: 0.06. Geomagic studio software (v2013, USA), Rhinoceros software (5.0, USA), Metlab Software (R2016b, USA). Desktop 3D printer independently developed by Zhejiang Key Laboratory of 3D Printing Technology and Equipment. Polylactic Acid (PLA) line (Shenzhen Eisendi technology co., LTD). Gel impression tray.

\section{METHODS}

\section{Take conjunctival sac gel impression.}

Sit the patient in a chair. The mold places the mixture in the syringe. Connect the die plate to the syringe. When the impression plate is placed over the eye, the patient is instructed to gaze down with the eye and the impression plate is inserted first into the fornix under the upper eyelid and then into the lower eyelid. Gently injection molding material, slow movement to avoid bubbles. Patients are asked to close their eyes so excess material can flow out through the perforation of the tray. While the mold is being taken, have the patient make eye movements, moving the eyes in the back. The impression material will solidify in two minutes. Take out the solidified gel model.

\section{Design 3D models of COPs.}

(a) Conduct 3D scanning of the conjunctival sac gel model. A binocular blue ray 3D scanner (as shown in Figure 1) was used to scan the conjunctival sac gel impression to obtain 3D point cloud data stored in .wrl format. In the software Geomagic studio, the point cloud was processed with noise removal and surface smoothing, etc., and the model of gel impression was reconstructed. (b) Model measurement: The Geomagicstudiov2013 software measured the 3D scanning reconstruction model of the conjunctival sac impression, including the gel model height $(\mathrm{mm})$, gel model thickness $(\mathrm{mm})$ and gel model base area $\left(\mathrm{mm}^{2}\right)$. (c) 3D model design: In Rhinoceros5.0 software, based on the original model, the surrounding contour was projected from the top view and adjusted, and the initially repaired bottom contour was obtained. Similarly, the contour is obtained from the side view projection (as shown in Figure 1). Combined with the shape of the finished prosthesis as a reference, the contour lines obtained from the above two steps were extracted and modified to obtain the bottom contour and the side contour after repair. Using the obtained contour line, the eye model is obtained by rotating sweep based on the contour line.

\section{3D printing of the COPs.}


Before 3D printing, the 3D finite element model of the personalized artificial eye designed above is first converted into $\mathrm{G}$ code in Slic3r24, and then passed into the desktop 3D printer (as shown in Figure 1). Set the filling density as $60 \%$ and the wall thickness as $1.6 \mathrm{~mm}$. The PLA were extruded (at $205^{\circ} \mathrm{C}$ ) by a heated metal nozzle $(0.4 \mathrm{~mm}$ in diameter), then moved horizontally and vertically, and deposited on the receiving table to form a prosthetic eye. This process was called as molten deposition manufacturing. The 3D printed white artificial eye is polished to make the surface smooth and the edge polished. Located the center of the pupil.

\section{Computer-aided quality assessment of COPs}

(a) Try on the COPs. The patients were tried on the white prosthesis to evaluate its function, and the palpebral fissure height, upper eyelid fullness, horizontal and vertical motion of the COP and the healthy eye were measured respectively. The traditional evaluation method is to manually conduct these measurements under the slit lamp with the corneal inverted spot as the target. In order to improve accuracy of measurement, we designed a computer-aided measurement program, which can accurately locate the center of the pupil. Then we evaluated the palpebral fissure height, upper eyelid fullness, horizontal and vertical mobility of prosthetic eyes and healthy eyes with the computer-aided measurement program.

(b) Assess the orbital fullness. Digital images of the lower head position of 5 patients were taken as shown in Figure 2(f), and the images were rotated and added with scales to compare the fullness between the reconstructed orbit with COPs and the healthy eyes. The evaluation was carried out by the way of doctors' and patients' scoring by questionnaire.

(c) Computer-aided assessment of the mobility of COPs. We developed a software for automatically analysising the mobility of COPs in Matlab R2016b software. For each patient, photos of different eye positions were taken, namely, the frontal, upper, lower, left, and right viewing positions. A circular sticker (red, $9 \mathrm{~mm}$ in diameter) was placed on each patient's forehead as a reference marker. Then these five images were input into the analysis software. During the image processing, the first step is to click randomly on the red circular marker, then on the right pupil and the left pupil. The edge detection algorithm was used to determine the area of the mark, the scale was calculated according to the actual size of the mark and the pixel width occupied, and the center point position of the mark was calculated automatically. The three points were denoted by $\mathrm{O}, \mathrm{L}$ and $\mathrm{R}$, and the rest positions are denoted by $\mathrm{O}$ ', $\mathrm{L}$ 'and $\mathrm{R}$ ' in the formula. Edge detection algorithm: Since the colors used by the markers are clearly distinguished from the facial colors, the RGB numerical ratio feature is used to directly define the edges and obtain the accurate edge of the markers. Determination of the diameter and center of the mark: After the edge of the mark area is obtained, the index difference of the horizontal X-axis is taken as the diameter of the mark, and the coordinate of the central point is the center of the circle:

$\mathrm{D}=x_{\max }-x_{\min }$ 


$$
\mathrm{O}=\left(\frac{\left(x_{\min }+x_{\max }\right)}{2}, \frac{\left(y_{\min }+y_{\max }\right)}{2}\right)
$$

The first calculation quantity: calculate the distance between the mark and the left and right pupil, using Euclidean distance:

$$
\begin{aligned}
& \text { Distance }_{\text {left }}=O L=\sqrt{\left(x_{L}-x_{0}\right)^{2}+\left(y_{L}-y_{0}\right)^{2}} \\
& \text { Distance }_{\text {right }}=O R=\sqrt{\left(x_{R}-x_{0}\right)^{2}+\left(y_{R}-y_{0}\right)^{2}}
\end{aligned}
$$

The second calculation quantity: the movement distance and Angle of the pupil in each direction: since the position of each picture is different when it is taken, the coordinate position on the different picture cannot be directly calculated. Therefore, the movement distance of the pupil in each direction can be calculated by taking the mark as the reference. Calculate the two vectors and do vector subtraction to get the vector (as shown in Figure 3). Vector length is calculated directly by modular operation, and the plane Angle of the vector is calculated by rad2deg (ATAN2 $(y, x))$ in Matlab R2016b software. The third calculation: the area of pupillary activity. Take the four extreme activity points of the left and right eyes as the vertex, and calculate the area of the mobilty (as shown in Figure 3 ).

D. Ethics approval and consent to participate: The study was conducted in accordance with the Declaration of Helsinki and approved by the Research Ethics committee of the Affiliated Eye Hospital of Wenzhou Medical University. Informed consent was obtained from all of participants before their inclusion in the study.

\section{lii. Results}

A. Sample collection. Patients were evaluated and those who met the conditions were included in the experiment. The basic information was shown in the table 1.

\section{Table 1. Patient information}




\begin{tabular}{|lllllll|}
\hline No. & Sex & $\begin{array}{l}\text { Age } \\
\text { (years) }\end{array}$ & Eye & $\begin{array}{l}\text { Reason for } \\
\text { enucleation }\end{array}$ & Implant & $\begin{array}{l}\text { Time for wearing ocular } \\
\text { prosthesis (years) }\end{array}$ \\
\hline 1 & Male & 49 & Right & Trauma & Yes & 4 \\
\hline 2 & Male & 26 & Right & Keratitis & Yes & 4 \\
\hline 3 & Female & 54 & Right & Trauma & Yes & 4 \\
\hline 4 & Male & 45 & Right & Trauma & Yes & 26 \\
\hline 5 & Male & 37 & Right & Trauma & Yes & 4 \\
\hline
\end{tabular}

\section{B. Design and Manufacture of COPs.}

1. 3D scanning results. The conjunctival sac gel impression was scanned by binocular blue - ray 3D scanner. The results showed that the binocular blue ray 3D scanner could reflect the conjunctival sac impression space structure with high fidelity (seen in Figure 1).

2. Computer-aided 3D reconstruction of COPs. The digital model of the gel impression was measured in the Geomagic Studio V2013 software. By removing some of the deformation error data of the gel itself and combining with the shape of the implant that is close to the results of the conjunctiva capsule of the patient, the accuracy of the design of the prosthesis was improved and the 3D model of the personalized prosthesis was reconstructed (seen in Figure 1).

3. 3D printing of COPs. 3D printing technology is used to produce semi-finished samples of personalized eye prosthesis (seen in Figure 1). After polishing the surface of the 3D printed prosthesis sample, the surface of the prosthesis is smooth and has good edge suitability with the conjunctival sac on the affected side.

\section{Quantitative evaluation of the function of COPs.}

The prosthesis was tried on in the conjunctival sac to evaluate the function of the prosthesis (see Figure 2). The contents include palpebral fissure height measurement of prosthesis and healthy eye, upper eyelid fullness measurement of prosthesis and healthy eye, and activity measurement of artificial eye.

1. Palpebral fissure height measurement of artificial eye and healthy eye. In Matlab R2016b software, the distance between upper eyelid margin and lower eyelid margin is measured by vertical line through the center of the pupil, as shown in Figure 2. Height of palpebral fissure of artificial eye and healthy eye was measured in 5 patients, and the measurement results were shown in Table 2. Independent sample t test was conducted on the data in software PRISM 6.0, and the results showed that the height of palpebral fissure in the artificial eye group was $0.8000 \pm 0.09529, \mathrm{~N}=5$. The height 
of palpebral fissure in the healthy eye group was $0.7660 \pm 0.1147, \mathrm{~N}=5$, and there was no statistical difference between the two groups ( $p>0.05$ ).

Table 2. Comparison of height between artificial eye and healthy eyelid fissure

\begin{tabular}{|c|c|c|c|c|}
\hline No. & $\begin{array}{l}\text { Palpebral fissure height of } \\
\text { COP\cm }\end{array}$ & $\begin{array}{l}\text { Palpebral fissure height of } \\
\text { healthy eye } \llbracket \mathrm{cm} \square\end{array}$ & Difference $\llbracket \mathrm{cm} \square$ & $\begin{array}{l}\text { Difference } \\
\text { errorlul }\end{array}$ \\
\hline 1 & 0.49 & 0.40 & 0.09 & 22.5 \\
\hline 2 & 1.07 & 1.00 & 0.07 & 7.0 \\
\hline 3 & 0.75 & 0.60 & 0.15 & 25.0 \\
\hline 4 & 0.79 & 0.95 & 0.16 & 16.8 \\
\hline 5 & 0.90 & 0.88 & 0.02 & 2.3 \\
\hline
\end{tabular}

2. Upper eyelid fullness evaluation of prosthesis and healthy eye. Digital images of the lower head position of 5 patients were taken, and the images were rotated and added scales, etc., to evaluate whether the fullness of the upper eyelid of the patients with artificial eye was consistent with that of the healthy eye (as shown in Figure 2). The evaluation was conducted by the way of doctors' and patients' scoring and evaluation, and the results were shown in Table 3.The artificial eye in the conjunctival sac had a good effect on the reconstruction of orbital fullness.

Table 3. Results of artificial eye and healthy eye fullness questionnaire

\begin{tabular}{|lll|}
\hline No. & Patients' self-reported & The doctor evaluation \\
\hline 1 & -1 & -1 \\
2 & 0 & 0 \\
3 & 0 & 0 \\
4 & 0 & 0 \\
\hline 5 & 0 & 0 \\
\hline Note: 0 means flat, negative means shrinking, positive means protruding \\
\hline
\end{tabular}


3. Activity measurement of COPs. Digital images of the patient's five eye positions were taken, as shown in Figure 3. In Matlab R2016b software, the activity of artificial eye and key eye is measured and compared (seen in Table 4 and Table 5). The activity range diagram is drawn (seen in Figure 3 ).

Table 4. Activity of artificial eye and healthy eye

\begin{tabular}{|c|c|c|c|c|c|c|c|}
\hline No. & Eye & $\begin{array}{l}\text { Upward } \\
\text { [cm! }\end{array}$ & $\begin{array}{l}\text { Downward } \\
\text { Dcm! }\end{array}$ & $\begin{array}{l}\text { Towards } \\
\text { the left } \\
\text { पcm! }\end{array}$ & $\begin{array}{l}\text { Towards the } \\
\text { right } \square \mathrm{cm} \square\end{array}$ & $\begin{array}{l}\text { Vertical } \\
\text { activity } \\
\square c m \square\end{array}$ & $\begin{array}{l}\text { Horizontal } \\
\text { activity } \text { पcm } \square\end{array}$ \\
\hline \multirow[t]{2}{*}{1} & $\mathrm{COP}$ & 0.17 & 0.57 & 0.32 & 0.23 & 0.74 & 0.55 \\
\hline & Healthy & 0.34 & 0.84 & 0.71 & 0.58 & 1.18 & 1.29 \\
\hline \multirow[t]{2}{*}{2} & $\mathrm{COP}$ & 0.15 & 0.48 & 0.17 & 0.24 & 0.63 & 0.41 \\
\hline & Healthy & 0.44 & 0.73 & 0.55 & 0.73 & 1.17 & 1.28 \\
\hline \multirow[t]{2}{*}{3} & $\mathrm{COP}$ & 0.46 & 0.11 & 0.17 & 0.07 & 0.57 & 0.24 \\
\hline & Healthy & 0.65 & 0.61 & 0.75 & 0.82 & 1.26 & 1.57 \\
\hline \multirow[t]{2}{*}{4} & $\mathrm{COP}$ & 0.43 & 0.63 & 0.56 & 0.31 & 1.06 & 0.87 \\
\hline & Healthy & 0.46 & 1.06 & 0.99 & 0.78 & 1.52 & 1.77 \\
\hline \multirow[t]{2}{*}{5} & $\mathrm{COP}$ & 0.13 & 0.46 & 0.32 & 0.04 & 0.59 & 0.36 \\
\hline & Healthy & 0.24 & 0.57 & 0.86 & 0.38 & 0.81 & 1.24 \\
\hline \multicolumn{8}{|c|}{ 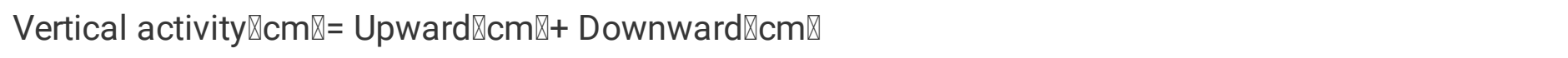 } \\
\hline
\end{tabular}

Table 5. Comparison of activity between artificial eye and healthy eye 


\begin{tabular}{|llll|}
\hline Activity & COP & Healthy & P \\
& $(\mathrm{cm} ;$ mean $\pm S D)$ & $(\mathrm{cm} ;$ mean $\pm S D)$ & \\
\hline Upward & $0.27 \pm 0.16$ & $0.43 \pm 0.15$ & 0.02 \\
\hline Downward & $0.45 \pm 0.20$ & $0.76 \pm 0.20$ & 0.01 \\
\hline Towards the left & $0.31 \pm 0.16$ & $0.77 \pm 0.16$ & 0.00 \\
\hline Towards the right & $0.18 \pm 0.12$ & $0.66 \pm 0.18$ & 0.00 \\
\hline Vertical activity & $0.72 \pm 0.20$ & $1.19 \pm 0.25$ & 0.00 \\
\hline Horizontal activity & $0.49 \pm 0.24$ & $1.43 \pm 0.23$ & 0.00 \\
\hline
\end{tabular}

\section{Iv. Discussion}

The main advantages of COPs are improved adaptability, mobility, and aesthetics. The major disadvantages include increased manufacturing time and cost. The core step of traditional acritical fabrication is to take the the impression of the conjunctival sac. There are many impression methods that exist. There are basic similarities between most techniques. Oculists who work with impression materials prefer non-reversible hydrocolloids with low viscosity and no irritation. Inject or transport the impression material into the recess and client looks straight ahead until the material is fixed. Then the impression is taken out, placed in the stone mold, and then the wax blank is made. Effectiveness and desirability of impression methods depend to a large degree on operator experience and equipment available.

In this study, we developed a semi-automatic design and fabrication workflow of COPs based on the new technique for conjunctival sac impression. The first step is to obtain a rough 3D model of outside surface of the conjunctival sac gel model with the 3D scanner. Second, the rough model is measured in three dimensions and main landmarks were extracted. Third, a COP model was constructed in the modeling software. COPs made by RE and 3D printing technologies can effectively make the COPs suitable for patients' conjunctival sac. This workflow improved the accuracy of the COPs, and also reduced patients' waiting time, which is worthy to be popularized and applied.

In addition, we also developed a software for automatically analyzing the mobility of COPs in Matlab R2016b software. In this software, we designed a computer-aided measurement program, which can accurately locate the center of the pupil. Compared with the traditional evaluation method, automatic analyzing software improved the accuracy of measurement and calculate the area of the mobility.

Our study still has some limitations and needs further improvement to achieve better practicality. Although a good impression technique can be expected to capture the internal tissue surface and fornix of the eye socket, most authors agree that attempts to wax test the eye prosthesis are important in 
assessing the appropriate eyelid opening and covered tissue profile. In order to solve this problem, it may be possible to quickly print various COPs by $3 \mathrm{D}$ printing technique, try it on, and choose the one that fits the best in the future.

\section{List Of Abbreviations}

COP Custom Ocular Prostheses

CAD Computer-aided design

CAM Computer-aided manufacture

RE Reverse Engineering

3D 3-Dimensional

FDM Fused Deposition

PLA Polylactic Acid

\section{Vi. Declarations}

Ethics approval and informed consent to participate: The study was conducted in accordance with the Declaration of Helsinki and approved by the Research Ethics committee of the Affiliated Eye Hospital of Wenzhou Medical University. Informed consent to participate in the study was obtained from all of participants.

Informed consent for publication: Informed consent to publish was obtained from all of participants before their inclusion in the study.

Availability of data and materials: The datasets used and analyzed during the current study are available from the corresponding author on reasonable request.

Competing Interests $\square$ None reported.

Funding $[$ The sponsor or funding organization had no role in the design or conduct of this research.

Authors' Contribution: Dr. SLJ had full access to all the data in the study and will take responsibility for the integrity of the data and the accuracy of the data analysis. Study concept and design: YX. Acquisition, analysis, or interpretation of data: YX, FW, QZB. Drafting of the manuscript: YX, WJ. Critical revision of the manuscript for important intellectual content: YX. Study supervision: SLJ.

Acknowledgements囚The authors acknowledge Wenzhou scientific research project (Y20190627). 


\section{Vii. References}

1. Amrith R. Ocular prosthetics: Use of a tissue conditioner material to modify a stock ocular prosthesis. The Journal of Prosthetic Dentistry. 1997.

2. Waggoner HW. OCULAR PROSTHETICS*. Clinical \& Experimental Optometry. 2010;49:133-45.

3. Ankita C. Iris Positioning Using a Grid Attached to a Spring Bow for a Custom Ocular Prosthesis. J Clin Diagn Res. 2017;11:ZD12-ZD3.

4. Mathews MF, Smith RM, Sutton AJ, Hudson R. The ocular impression: A review of the literature and presentation of an alternate technique. Journal of Prosthodontics Official Journal of the American College of Prosthodontists. 2010;9:210-6.

5. Chiramana S, Anne G, Ravuri K, Boppana PP, Appana KC. A Time Saving Method to Fabricate a Custom Ocular Prosthesis. Journal of Orofacial Research. 2013;3.

6. Raizada K, Rani D. Ocular prosthesis. Contact Lens \& Anterior Eye. 2007;30:152-62.

7. John AV, Anilkumar S, Rajesh C, Raghavan S. A novel technique of custom ocular prosthesis fabrication. Journal of Oral Research and Review. 2016;8:82-.

8. JaeSang, Hyun S, Kim, Woon S, Baek, Min K, et al. Semi-automated fabrication of customized ocular prosthesis with three-dimensional printing and sublimation transfer printing technology. Scientific reports. 2019.

9. Shankaran G, Deogade SC, Dhirawani R, Professor. Fabrication of a Cranial Prosthesis Combined with an Ocular Prosthesis Using Rapid Prototyping: A Case Report. Journal of Dentistry. 2015;13:6872.

10. Sally, Beiruti, Anne-Claire, Le, Henaff, Arjun, et al. A novel, efficient 3D-printing based manufacturing process for custom ocular prostheses. Conference proceedings: Annual International Conference of the IEEE Engineering in Medicine and Biology Society IEEE Engineering in Medicine and Biology Society Annual Conference. 2019;2019:3734-7.

11. Ye X, Wang S, Zhu Y, Shao H, Lou L, Qian D, et al. Automatic Design and Fabrication of a Custom Ocular Prosthesis using 3D Volume Difference Reconstruction (VDR). IEEE Access. 2018:1-.

\section{Figures}



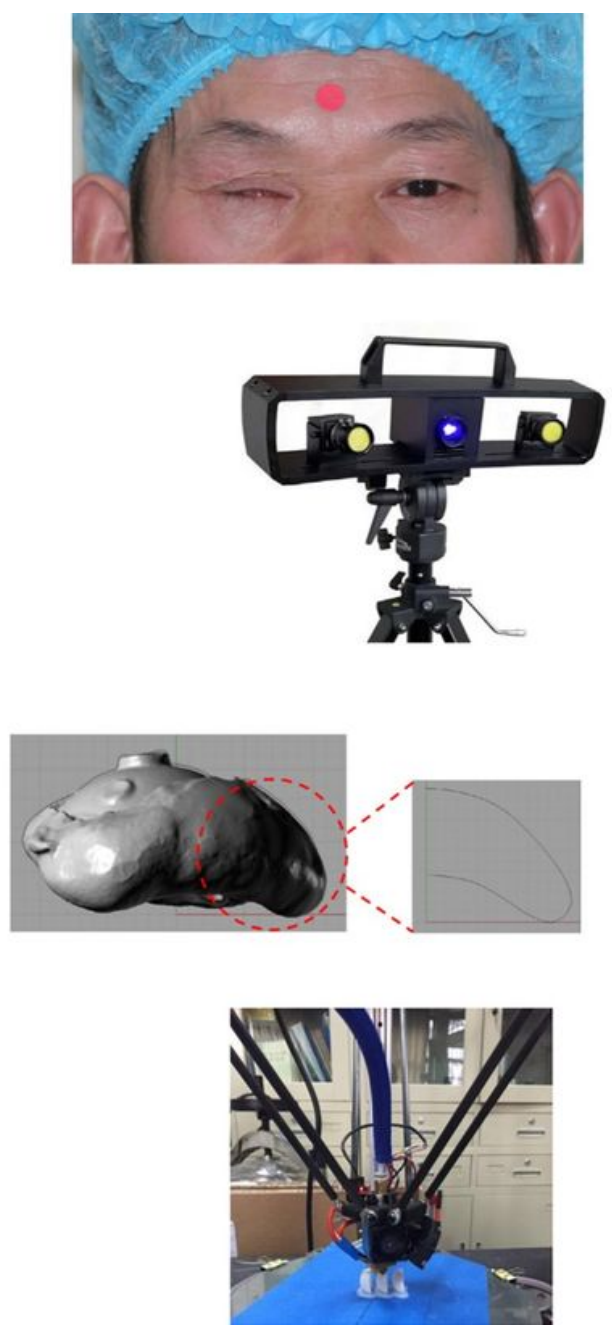

Figure 1

Workflow of Semi-automatic 3D printing of personalized prosthesis (patient No.1) 


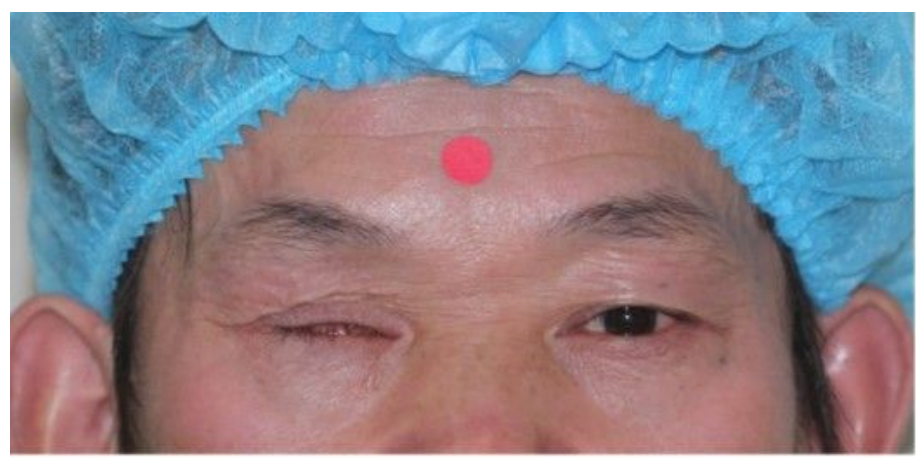

(a)

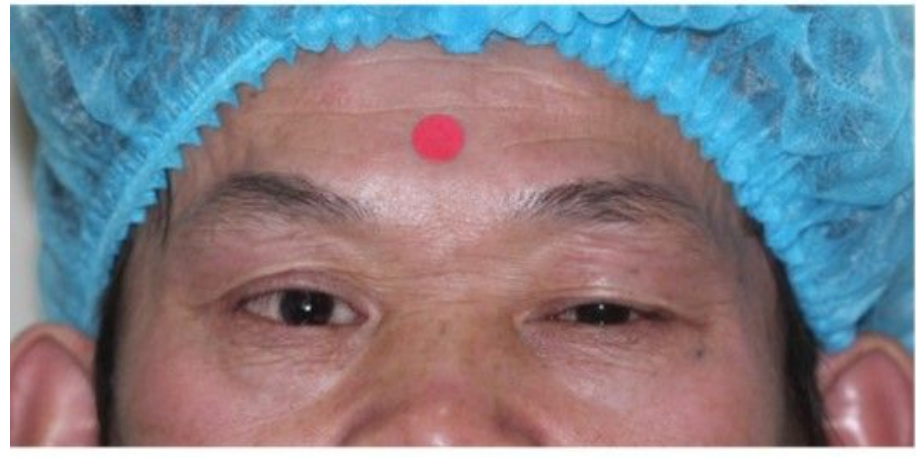

(c)

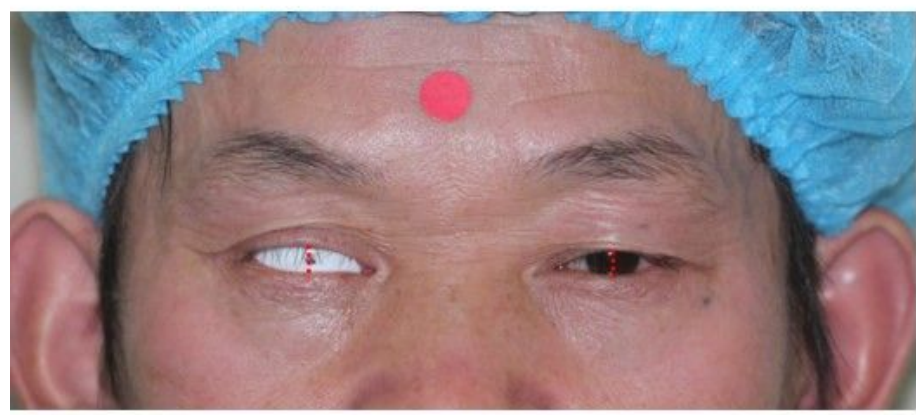

(e)

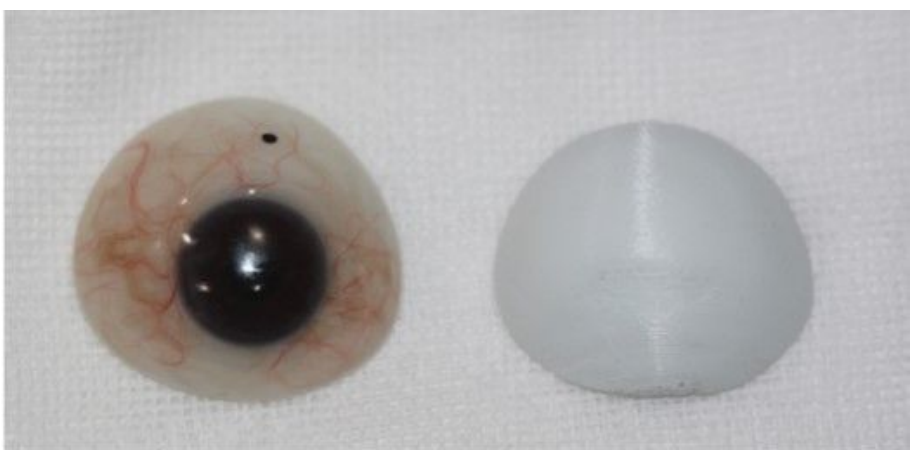

(b)

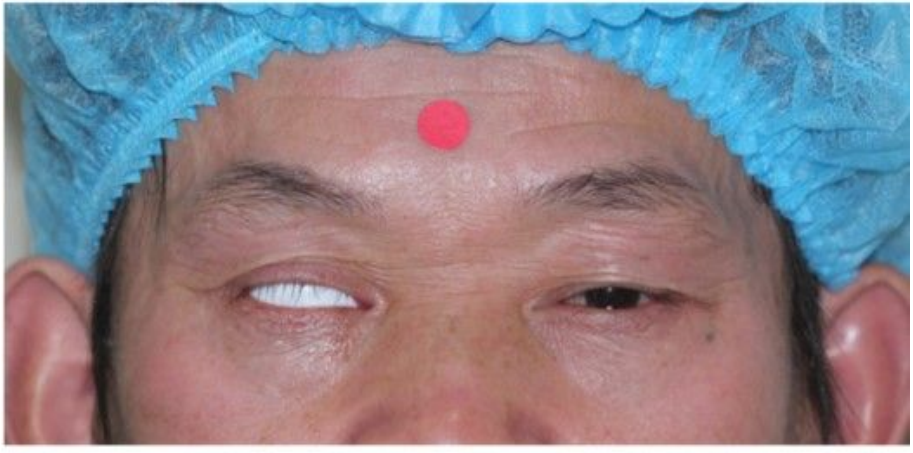

(d)

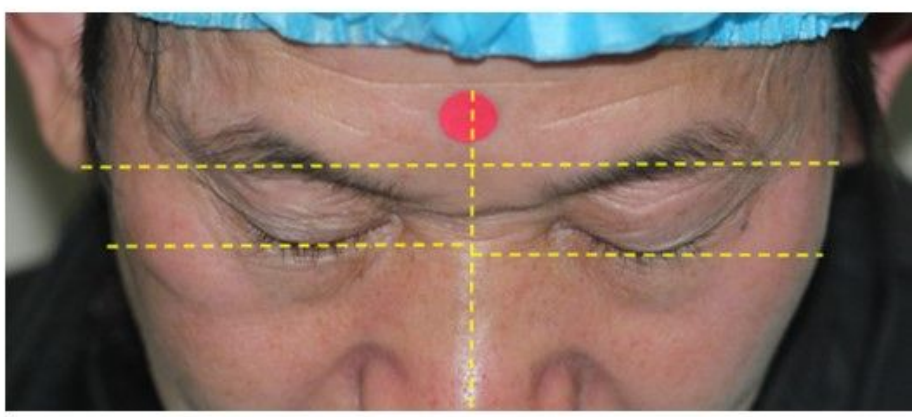

(f)

\section{Figure 2}

Try on the prosthesis (Patient No. 1): (a) Male, 49 years old, right eye after enucleation for 4 years; (b) a traditional hand-made ocular prosthesis (left) and a 3D printed custom ocular prosthesis (right); (c) Patient wearing a traditional hand-made ocular prosthesis; (d) Patients wear 3D printed custom ocular prosthesis (without painting); (e) Schematic diagram of palpebral fissure height measurement; (f) Evaluation of upper eyelid fullness of custom ocular prosthesis and healthy eye. 

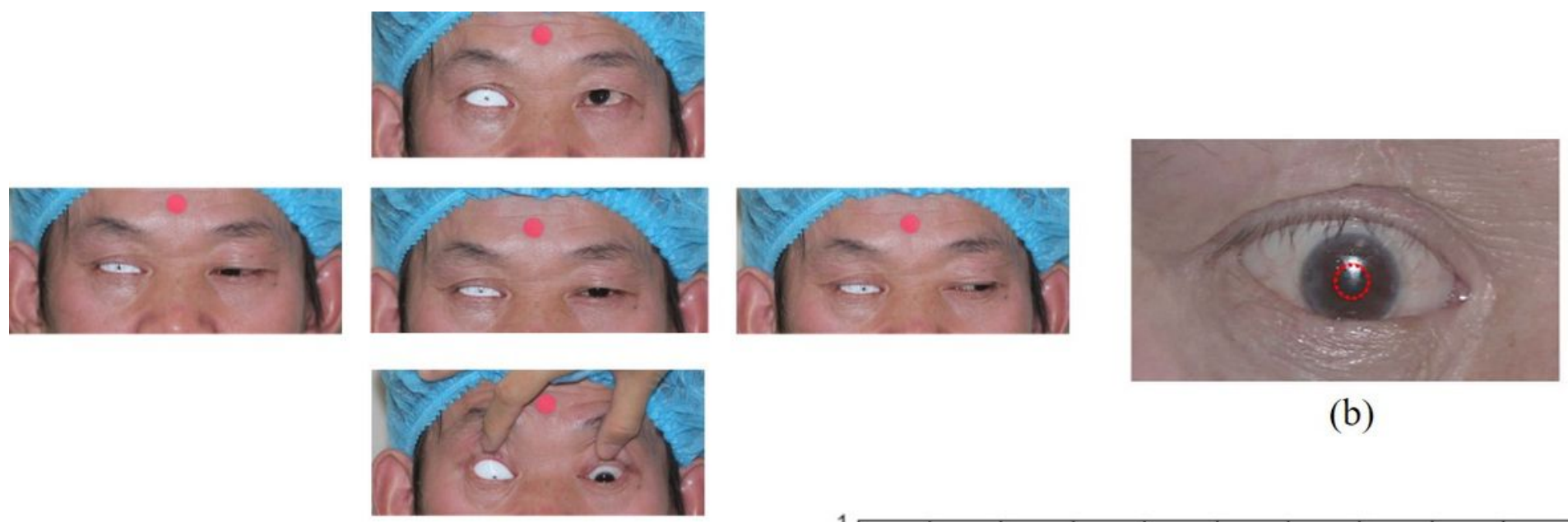

(b)

(a)
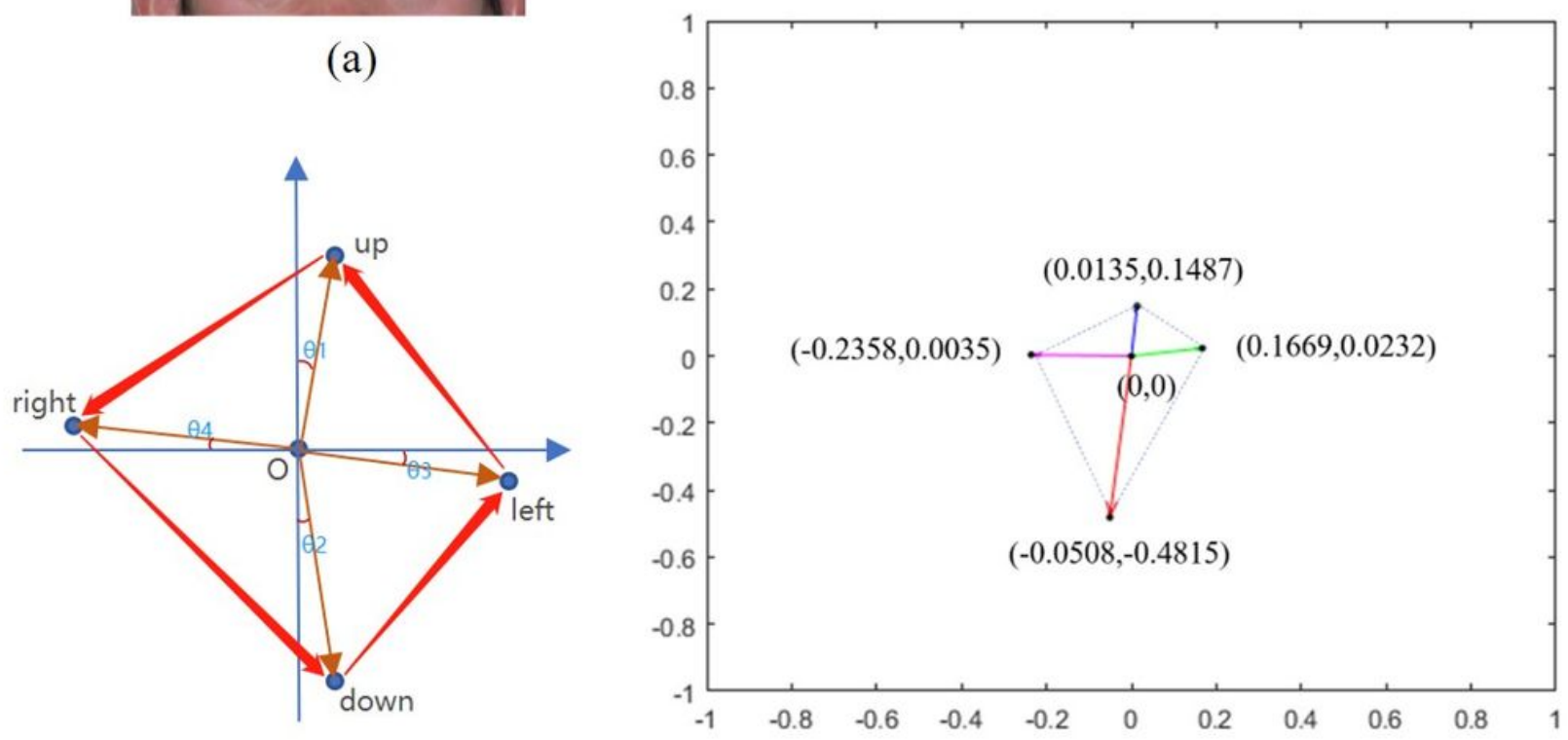

(c)

(d)

\section{Figure 3}

Activity assessment of the artificial eye and healthy eye: (a) The patient wore the custom ocular prosthesis in 5 positions of eye: the emmetropia, upward position, downward position, towards the left, and towards the right; (b) Considering the differences between the corneal reflex point of custom ocular prosthesis and healthy eye, a computer-aid method for locating the center of the pupil is used and the red dotted line circle represents the pupil; (c) Schematic diagram of computer-aid calculation of pupil activity; (d) Schematic diagram of computer-aid calculation of pupillary activity area. The coordinates of the center of the pupil of the right eye were $(0,0),(0.0135,0.1487),(-0.0508,-0.4815),(0.1669,0.0232)$, and $(-0.2358,0.0035)$ in the emmetropia, upward position, downward position, towards the left, and towards the right positions. The activity area is $0.1073 \mathrm{~cm} 2$. 